\title{
Design of an Embedded Hardware for Motor Control of a High Performance Electric Vehicle
}

\author{
Andrés M. Asprilla ${ }^{1}$, Wilmar H. Martinez ${ }^{1}$, Luis E. Muñoz ${ }^{2}$, Camilo A. Cortés ${ }^{1}$ \\ ${ }^{1}$ Universidad Nacional de Colombia \\ ${ }^{2}$ Universidad de los Andes \\ Bogotá - Colombia \\ amavaldes@inf.ufrgs.br, whmartinezm@unal.edu.co, lui-muno@uniandes.edu.co, caacortesgu@unal.edu.co
}

\begin{abstract}
In current automotive applications, novel and robust communication technologies have been used to optimize some features related to security, fuel consumption and user interface. However, the automotive communication protocols present two problems: 1 . the connections between devices are very complex and their maintenance is difficult, and 2 . most of the commercial devices, specialized to send data between devices, use proprietary software to perform operations inside the vehicle. In this work, a novel embedded platform was designed to measure and control the speed and torque of electric motors of a $200 \mathrm{~kW}$ electric vehicle. It is a versatile, modular and opensource design, which exchanges data with each motor using the CAN Bus protocol to control their speed. Also, the implementation and maintenance of the proposed platform becomes simpler. The proposed platform was built and tested with the devices that are used inside the car.
\end{abstract}

Keywords- Field Buses, Electric Vehicles, CAN Bus, Embedded Systems, GNU/Linux, SocketCAN, Instrumentation, DC Motors, Ultracapacitors, Single Board Computers.

\section{INTRODUCTION}

Electric Vehicles (EVs) are an improved, alternative and emerging technology with a growing interest due to the environmental issues generated by the fuel consumption and greenhouse emissions in transportation systems. However, these vehicles demand highly efficient, compact and highpower converters to give them enough torque and speed to the daily requirements of the users, especially, in places with varying topography and unstable soils [1]. Electric automotive technologies have gained a great interest because EVs are promising in terms of the environmental impact caused by the emissions caused by Internal Combustion Engine (ICE) vehicles [2].

However, in comparison with ICE vehicles, EVs present some disadvantages: most of them have lower torque and speed, and they cannot be used to go over long distances because of the reduced energy capacity of the storage devices [3]-[4]. In order to study and promote the use of EVs, Universidad Nacional de Colombia and Universidad de Los Andes have been working on a project with the objective of designing and constructing a high performance EV (a dragster) capable to run a quarter mile in 10 seconds [6].

The purpose of this electric dragster is to provide a demonstrative platform of the EVs capabilities. Based on the energy and power calculation and a sizing methodology presented in [6] and [7], six ultracapacitors of $125 \mathrm{~V}$ and $62 \mathrm{~F}$ were chosen to store $600 \mathrm{Wh}$ of electric energy. This storage setup was chosen because it provides a high power density, which is necessary to supply the motors for a short period of time, while the vehicle is accelerating and reaches the end of the race [5].

In addition, four $50 \mathrm{~kW}$ brushless permanent magnet motors (BLPM) with a peak torque of $440 \mathrm{Nm}$ were selected to fulfill the needed power and torque of the race. Fig. 1 shows the schematic diagram of the dragster vehicle.

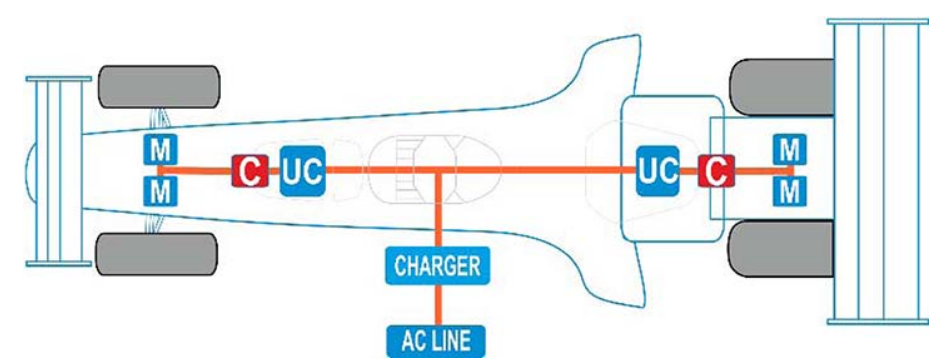

Fig. 1. High performance electric vehicle schematic diagram. $(M=$ Motor, $C=$ DC-DC Converter, UC = Ultracapacitors).

For this project, the robustness and the communication of all modules in the vehicle are important features that need to be considered to achieve a high level of reliability in the vehicle. As a matter of fact, current vehicles include new technologies that are useful in terms of security, stability and passenger comfort (e.g. Navigation systems, entertainment systems, etc.). Therefore, these systems need to be connected and exchange messages in a decentralized form [8]. For this reason, field buses are used to decentralize the communication and to reduce the number of required wires to implement a control system [9].

In this way, one of the most used protocols in the automotive industry is the CAN Bus, created in the 80 s by BOSCH Corporation. It is a serial and multi-node protocol, widely used in distributed systems due to its high reliability, multicasting and high performance under heavy load networks. Therefore, for the project development, considering the advantages of using the CAN protocol, this protocol was chosen because all the devices used in the vehicle are compatible with it, and the protocol offers the needed flexibility, speed, robustness and scalability to carry on the communication between all modules [10]. 
As seen in Fig. 1, the vehicle has four independent motors controlling each wheel separately. This approach needs an efficient command system that can send the CAN Bus commands appropriately to each motor. Thus, an embedded system is required to efficiently solve this problem and could be used as a command and measuring system for the proposed electric vehicle.

Consequently, a control circuit board is needed to manage all the devices in the vehicle. However, most of the CAN Bus controllers and messaging systems are expensive, and adapt them to the needs of this project is a complex task. For that reason, to customize the communication protocols to be usable by all the devices, it was necessary to create a novel platform that uses open-source software and hardware designs. The use of a customizable platform offers the advantage of scalability and flexibility to future needs of the project.

Thereby, an embedded platform loaded with the GNU/Linux Operating System was designed to send messages through the bus using the SocketCAN interface, which offers the necessary flexibility to set-up the communication interface [10]. The developed board offers the novelty of using opensource software and hardware designs. In addition, all the hardware and software designs used to develop the protocol are modifiable and future designers can use the developed software and hardware to add new features. Finally, GNU/Linux operating system offers more alternatives that are useful to support new communication protocols such as USB, and TCP/IP protocols to transfer information via Wi-Fi or Ethernet during the race. The development of the circuit boards is under creative commons license (CC-BY-SA) and it was done in the electrical and electronic engineering department of the Universidad Nacional de Colombia [11].

Moreover, this topic has not been considered in the state of the art, and there is a lack of contributions where platforms that run GNU/Linux operating are used to manage CAN Bus messages to control and measure the vehicle variables. In this work, the proposed communication mechanism was tested, and it is demonstrated that the developed architecture is fully functional and offers the expected levels of reliability needed for the application.

\section{ELECTRIC DRAGSTER}

To reach the quarter mile in 10 seconds, four $50 \mathrm{~kW}$ brushless permanent magnet motors with a peak torque of 440 $\mathrm{Nm}$ were chosen to fulfill the needed power and torque to reach the race. These motors demand high levels of voltage and current along the race. Therefore six 62F $125 \mathrm{~V}$ Ultracapacitors are required to supply the required energy density. Since the race duration is short, the energy delivered by the capacitors is enough to give the required energy during the race interval.

As Fig. 1 shows, the electric system is divided into two parts: two motors on the frontal side and two motors on the back side controlling each wheel independently. Each pair is fed by three ultracapacitors.

\section{A. Electric Motors}

The electric motors chosen for the application were the PowerPhase50 manufactured by UQM Technologies. They offer a maximum power of $50 \mathrm{~kW}$ (200 kW total). In addition, as each motor will be connected to each wheel, each motor has an inverter that is fed with 300 VDC obtained from a DC-DC converter which is supplied by the ultracapacitors. The supply voltage needs to be sampled and transmitted via TCP/IP protocol for further analysis. Fig. 2 a) shows the selected motor and its three-phase inverter. The inverter provides a control module, which is capable of controlling the motor through a potentiometer or using the CAN Bus protocol at $125 \mathrm{kbps}$. Considering that four motors have to be controlled at the same time, it is necessary to have a low-latency bus in order to send the information to each motor efficiently. Each motor supports three control modes: speed (with torque limit), torque (with speed limit) and voltage (with torque limit).

\section{B. Ultracapacitors}

The ultracapacitors selected for this application are the $62 \mathrm{~F}$ and $125 \mathrm{~V}$ manufactured by NESSCAP. Each module can handle a peak current of $1800 \mathrm{~A}$, and can store $134.5 \mathrm{Wh}$ of energy. Fig. 2 b) shows the selected ultracapacitor. Each module contains forty-eight $3000 \mathrm{~F} 2.7 \mathrm{~V}$ large cylindrical ultracapacitor cells. Also, the module has a negative temperature coefficient (NTC) resistor to measure its internal temperature. In addition, it has three DC fans, operating at 24 VDC, which reduce the internal temperature. Finally, it is important to mention that this ultracapacitor does not include a CAN Bus monitoring circuit, therefore a "daughter" board design was developed to measure and monitor the voltage and temperature of each module and report the measured data.

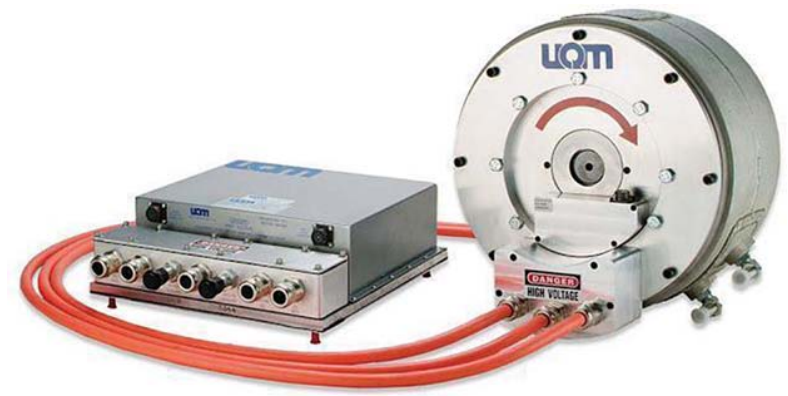

(a) UQM PowerPhase 50kW Electric motor

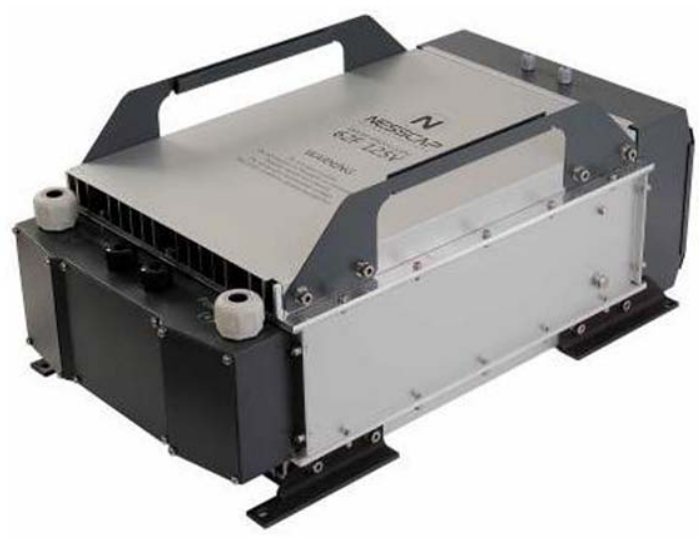

(b) Nesscap 62F / 125 V Capacitor

Fig. 2. The electric motor and ultracapacitor used in the dragster 


\section{ELECTRONIC CONTROL AND SUPERVISION UNIT (ECU)}

A typical EV uses different sensors and circuits to measure the stored energy and speed to have accurate data, which is used to optimize the efficiency of the vehicle. The information captured through those sensors is collected by digital embedded systems and needs to be processed. The complexity of those operations is becoming higher as the features of the vehicle increase, needing more powerful processing units capable of performing those operations. For that reason, the embedded hardware and software used in electric vehicles contribute about 30 to 40 percent of its total cost [12].

Fig. 3 shows a typical electric vehicle setup, which contains different sensors, actuators, control and supervision modules, which are used to send and process data captured during the race.

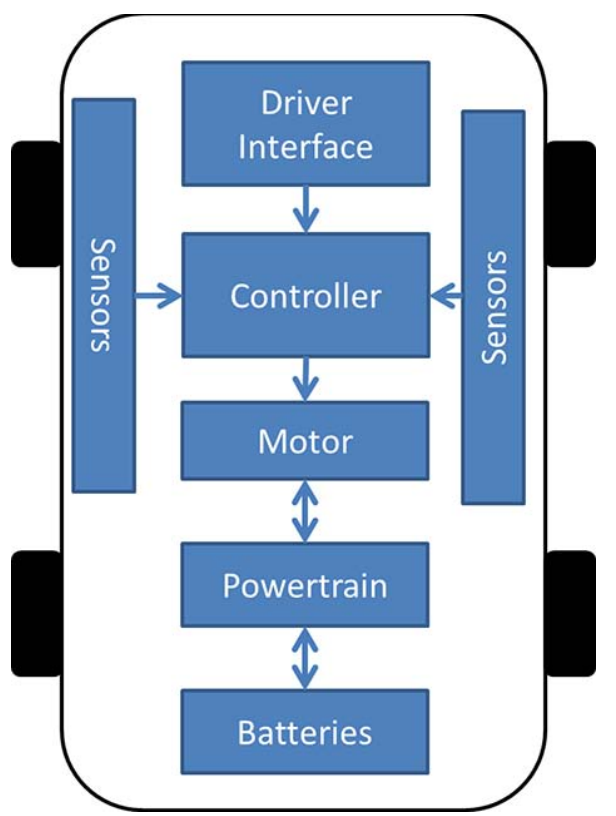

Fig. 3. An electric vehicle standar setup.

As seen in Fig. 3. A high quantity of wires is required to connect all the peripherals and sensors used to control the different variables used in the vehicle. In order to reduce the quantity of wires, decentralized communication protocols such as the CAN Bus is used to connect all the sensors to the supervision unit. With bus communication topologies, vehicular wiring can be reduced by 75 percent [14] [15].

Actually, there are some supervision units in the market, such as the MotoHawk ${ }^{\circledR}$ PCM-112, Raptor ${ }^{\circledR}$ HCM-90 or Raptor GCM-196 [13]. However, those units are expensive and its internal firmware is limited, and cannot be modified in order to add new features, also, they need an extra module to save and report the measured data to be processed and to save the race statistics. For that reason, a new embedded platform is proposed and developed with the objective of being customizable, modular and capable of supporting the desired features. Table I shows a comparison between the analyzed supervision platforms.

TABLE I. MEASURED AND SENT COMMANDS TO THE ELECTRIC MOTOR

\begin{tabular}{|c|c|c|c|c|}
\hline Feature & PCM-112 & HCM-90 & GCM-196 & Developed \\
\hline Processor & $\begin{array}{c}\text { MPC5644A, } \\
120 \mathrm{MHz}\end{array}$ & $\begin{array}{c}\text { MPC5534, } \\
80 \mathrm{MHz}\end{array}$ & $\begin{array}{c}\text { TC1793, } \\
260 \mathrm{MHz}\end{array}$ & $\begin{array}{c}\text { i.MX233, } \\
454 \mathrm{MHz}\end{array}$ \\
\hline RAM & $192 \mathrm{kB}$ & $64 \mathrm{kB}$ & $256 \mathrm{kB}$ & $128 \mathrm{MB}$ \\
\hline Flash & $2 \mathrm{MB}$ & $2 \mathrm{MB}$ & $4 \mathrm{MB}$ & $1 \mathrm{~GB}$ \\
\hline USB & 0 & 0 & 0 & 2 \\
\hline UART & 1 & 1 & 1 & 1 \\
\hline
\end{tabular}

As seen in Table 1, the developed supervision system has more features and offers more communication protocols such as the USB, which offers flexible capabilities and can support a variety of peripherals.

\section{PROPOSED COMMAND AND MEASURING SYSTEM}

To command the electric motors, an embedded platform was designed to send and receive the required information of the different connected nodes of the vehicle using the CAN Bus communication protocol.

To perform the measuring and command operations, different hardware and software components were designed and tested to have an accurate control of each variable of the motor. The motors are commanded by using the speed mode (with torque limit) and can support the torque mode (with speed limit).

The master board uses the NXP ARM926 i.MX233 applications processor. It has a variety of external interfaces such as UART, SPI, USB 2.0 and boots Linux through an SD card interface and has a 64 MB DDR SDRAM. Also, the embedded platform contains an ARM Cortex M4, that can act as a co-processing unit. The development board is based on a free hardware design. It can be modified and reproduced under the Creative Commons license [11]. Fig. 4 shows the board setup, indicating its main components.

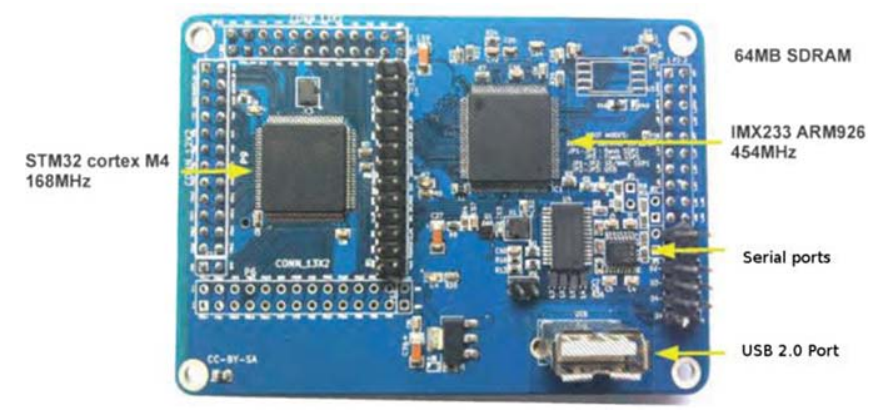

Fig. 4. i.MX233 STAMPS board [11]

As the i.MX233 SoC does not support the CAN Bus controller peripheral, it was necessary to develop an auxiliary 
board which includes the necessary hardware to support that protocol. To extend the CAN Bus interface support to the embedded platform, the Microchip's MCP2515 IC was used. It is connected to the SPI interface of the main SoC, enabling the DMA access to have a low-latency response despite of the used operating system. It is important to mention that the Linux SocketCAN interface supports the selected CAN Bus driver IC, reducing the required software to control the interface. Fig. 5 shows a picture of the developed board, showing its main important features. Also, the board shown in Fig. 5 contains a DC-DC converter, which gives the required supply voltage to the embedded platform shown in Fig. 3 and to extend the CAN Bus communication capabilities to the main embedded system board.

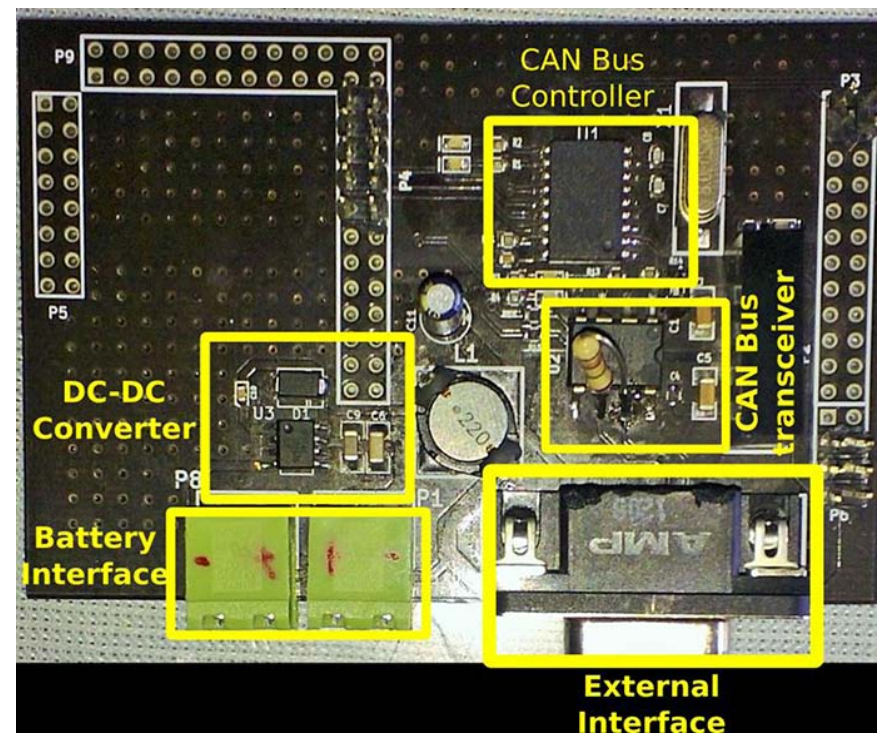

Fig. 5. Communications auxiliary board

Figs. 4 and 5 show the developed communications interface used to send commands to the motor controller using GNU/Linux as the main OS and the CAN Bus as the main network communications interface. The advantages of the proposed architecture are: first, the hardware and software used to communicate with the motors are open-source and can be used as an external communications interface to send data using data protocols such as Wi-Fi or GPRS. Second, the system is reliable and can be used as a communications interface to connect other CAN Bus automotive peripherals such as navigation or reference systems. It is well-known that GNU/Linux is not a real-time operating system, so, the installed kernel and the root file system were optimized in order to use only a few modules and services, necessary to run the developed software.

\section{TESTS AND RESULTS}

In order to check the functionality of the control system, different tests were performed to show the accuracy of the control and measuring system. Different speeds were set and transferred to the motor and the response was measured using an RPM meter coupled to the motor axis. Figs. 6 a) and 6 b) show the used test bench to test the electric motors and Fig $6 \mathrm{c}$ ) shows the CAN Bus waveforms sent to the motor to test the revolutions.

Table II shows a comparison between the speed commands sent to the motor using CAN Bus messages and the real speed response received from the motor at no-load condition.

TABLE II. MEASURED AND SENT COMMANDS TO THE ELECTRIC MOTOR

\begin{tabular}{|c|c|c|}
\hline $\begin{array}{c}\text { CAN Bus Speed } \\
\text { (RPM) }\end{array}$ & $\begin{array}{c}\text { Measured Speed } \\
\text { (RPM) }\end{array}$ & $\begin{array}{c}\text { Motor Voltage } \\
\text { (V) }\end{array}$ \\
\hline 400 & 402 & 286.4 \\
\hline 800 & 798 & 294.6 \\
\hline 1200 & 1198 & 293.5 \\
\hline
\end{tabular}

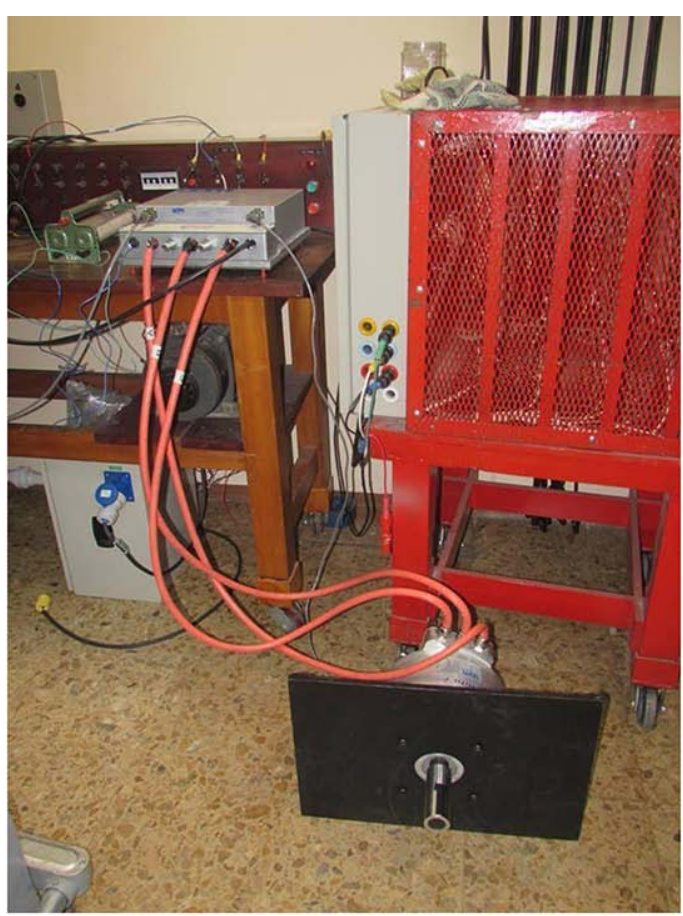

(a) Motor and controller setup

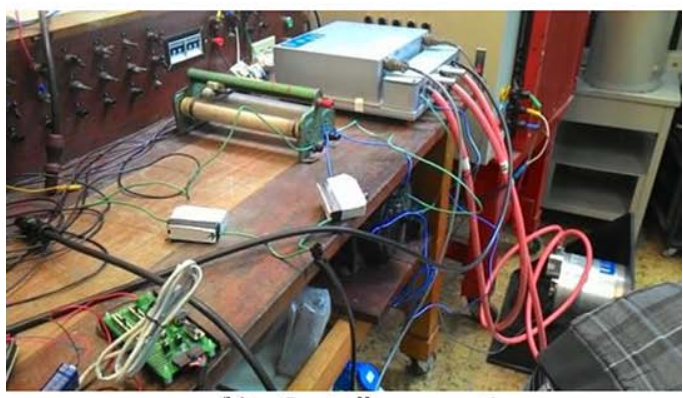

(b) Controller connections 


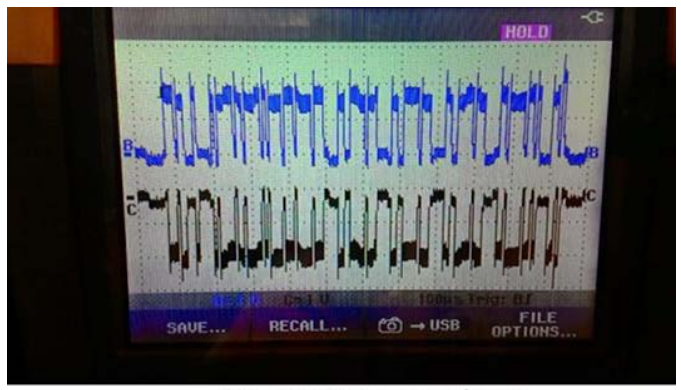

(c) CAN Bus waveforms

Fig 6. Test bench and CAN Bus communication waveforms

\section{CONCLUSION}

This work shows how the embedded platform for controlling electric motors has been developed. The system sets a desired speed and can measure the supply voltage of the electric motors. To test the system, a no-load test was conducted. The BLPM motor was supplied with $300 \mathrm{~V}$ DC and the embedded system was connected to the motor using the CAN Bus communications protocol. The system worked properly and the motor rotates at the desired speed, as seen in Table I. Therefore, the functionality of the designed platform was validated. It is evident that free software tools such as the GNU/Linux OS and the SocketCAN API can control the electric motors employed in this dragster, however, it needs some optimizations to reduce the jitter and unpredictable delays caused by a non-real-time operating system. The platform, designed by the authors is an open-source design, which could be used in other electric mobility projects. The platform source code could be available upon request. Finally, it could be concluded that using the CAN bus protocol, it is possible to reduce the number of required wires to control the electric motors, and to report the race information.

\section{REFERENCES}

[1] M. Yilmaz and P. T. Krein, "Review of Battery Charger Topologies, Charging Power Levels, and Infrastructure for Plug-In Electric and Hybrid Vehicles," in IEEE Transactions on Power Electronics, vol. 28, no. 5, pp. 2151-2169, May 2013.

[2] Ping Guo and Peng Liu, "Research on development of electric vehicles in China," 2010 International Conference on Future Information Technology and Management Engineering, Changzhou, 2010, pp. 94-96.

[3] K. Katsura and M. Yamamoto, "Optimal stability control method for transformer-linked three-phase boost chopper circuit," 2012 IEEE Energy Conversion Congress and Exposition (ECCE), Raleigh, NC, 2012, pp. 1082-1087.

[4] W. Martinez, M. Yamamoto, J. Imaoka, F. Velandia and C. Cortes, "Efficiency Optimization of a Two-Phase Interleaved Boost DC-DC Converter for Electric Vehicle Applications,” IEEE International Conference on Power Electronics - IPEMC 2016-ECCE Asia, pp. 1-7, 2016.

[5] Y. Cheng, R. Trigui, C. Espanet, A. Bouscayrol and S. Cui, "Specifications and Design of a PM Electric Variable Transmission for Toyota Prius II," in IEEE Transactions on Vehicular Technology, vol. 60, no. 9, pp. 4106-4114, Nov. 2011.

[6] W. Martinez, C. Cortes, L. Munoz and M. Yamamoto, "Design of a 200 kW Electric Powertrain for a High Performance Electric Vehicle", in Ingenieria e Investigacion Journal, vol. 36. no. 3, pp. 66-73, 2016.

[7] W. Martinez, C. Cortes and L. Munoz, "Sizing of ultracapacitors and batteries for a high performance electric vehicle," 2012 IEEE International Electric Vehicle Conference, Greenville, SC, 2012, pp. 1-6.
[8] W. Martinez and C. Cortes, "Design a DC-DC Converter for a High Performance Electric Vehicle," 2012 International Conference on Connected Vehicles and Expo (ICCVE), Beijing, 2012, pp. 335-340.

[9] Shi Shengbing, Song Chunyan and Wu Yanlin, "CAN bus performance test technology," 2015 12th IEEE International Conference on Electronic Measurement \& Instruments (ICEMI), Qingdao, China, 2015, pp. 13951399.

[10] F. Velandia, W. Martinez, C. Cortes, M. Noah and M. Yamamoto, "Power Loss Analysis of Multi-Phase and Modular Interleaved Boost DC-DC Converters with Coupled Inductor for Electric Vehicles," IEEE 18th European Conference on Power Electronics and Applications, EPE'16-ECCE Europe, pp. 1-9, 2016.

[11] Z. Qi, Ping Dong, K. Ma and N. Sargeant, "A design of in-car multilayer communication network with Bluetooth and CAN bus," 2016 IEEE 14th International Workshop on Advanced Motion Control (AMC), Auckland, New Zealand, 2016, pp. 323-326.

[12] M. Sojka, P. Píša, M. Petera, O. Špinka and Z. Hanzálek, "A comparison of Linux CAN drivers and their applications," International Symposium on Industrial Embedded System (SIES), Trento, 2010, pp. 18-27.

[13] i.MX233 STAMP Repository: https://github.com/Linux-enCaja/stamps.

[14] CHAKRABORTY, Samarjit, et al. Embedded systems and software challenges in electric vehicles. Proceedings of the Conference on Design, Automation and Test in Europe. EDA Consortium, 2012. p. 424-429.

[15] New Eagle ECU Selection Matrix, Source: https://www.neweagle.net/support/wiki/images/archive/e/e6/201410021 53545!Raptor ECU Selection.pdf

[16] Abbott-McCune, S., \& Shay, L. A., Intrusion prevention system of automotive network CAN bus. In Security Technology (ICCST), 2016 IEEE International Carnahan Conference on (pp. 1-8). IEEE.

[17] AC Delco, "CAN Communication," TechConnect, vol. 16, no. 1, pp. 13, Jan 2009. 